\title{
Interband absorption and its relation to the electronic structure of $\mathrm{Co}-\mathrm{Cr}$ solid solutions
}

\author{
V.M. Bondar, V.S. Stashchuk, O.P. Polianska, V.G. Kudin, A.O. Statsenko \\ Taras Shevchenko Kyiv National University, Physics Department, Chair of Optics, \\ 64, Volodymyrska str., Kyiv 01601, Ukraine \\ E-mail: bond270587@i.ua
}

\begin{abstract}
The refraction $n$ and absorption $\chi$ indices of paramagnetic $\mathrm{Co}-\mathrm{Cr}$ alloys with 10,20 and $35 \%$ chromium concentrations were measured within the spectral range 0.23 to $2.8 \mu \mathrm{m}(0.44 \ldots 5.36 \mathrm{eV})$ at room temperature by spectral ellipsometric methods. Based on these data, the dielectric permittivity $\varepsilon$, optical conductivity $\sigma$ and reflection coefficient $R$ at normal incidence were calculated. The dispersion of optical conductivity $\sigma(h v)$ and dispersion curves of residual optical conductivity $\Delta \sigma(h v)=\sigma(h v)-(1-\mathrm{c}) \times$ $\times \sigma_{\mathrm{Co}}(h v)$ were analyzed in detail $\left(c-\mathrm{Cr}\right.$ impurity concentration, $\sigma_{\mathrm{Co}}(h v)-$ optical conductivity of Co solvent). It has been ascertained that new impurity bands are formed in the electronic spectrum of $\mathrm{Co}$ solvent when $\mathrm{Cr}$ impurities are added, which are responsible for the extra absorption in $\mathrm{Co}-\mathrm{Cr}$ alloys. The localization of impurity energy levels in Co-Cr alloys at 1.8 ...2.0 eV above the Fermi level has been suggested.
\end{abstract}

Keywords: Co-Cr alloy, electronic structure, impurity band, energy subband, electron density, interband transition, density of electron states, optical conductivity spectrum.

Manuscript received 15.10.12; revised version received 28.11.12; accepted for publication 26.01.13; published online 28.02.13.

\section{Introduction}

As engineering practice shows, 3d-transition metal alloys are rather promising for using them in modern electromagnetic devices [1]. In particular, cobalt-based materials due to their exceptional magnetic properties have been widely used in systems with magnetic recording media [2]. Of particular interest is the study of magnetic properties of ferromagnetic alloys of cobalt and antiferromagnetic chromium, as while adding $\mathrm{Cr}$ the magnetic moment of $\mathrm{Co}-\mathrm{Cr}$ alloys decreases sharply and reaches zero at about $15 \%$ of $\mathrm{Cr}$ content [3]. In addition, these alloys are characterized by extremely high corrosion resistance, and so are used as functional materials in corrosive environments.

Although experimental and theoretical properties of the basic elements of the Co-Cr alloy - cobalt and chromium - are so far studied in detail [4-6], the wide practical application of these alloys is limited by lack of information about their electronic structure that determines their various physical properties. Therefore, a research of optical properties and electronic structure of $\mathrm{Co}-\mathrm{Cr}$ alloys was carried out in this work by using the high-precision spectral-ellipsometric study.

\section{Experimental procedure}

Optical properties of polycrystalline $\mathrm{Co}-\mathrm{Cr}$ alloys were studied at room temperature on samples obtained by repeated vacuum arc melting of pure $\mathrm{Co}$ and $\mathrm{Cr}$ with appropriate mass ratios in an argon atmosphere. To achieve better uniformity, the samples were annealed in the same atmosphere at $900^{\circ} \mathrm{C}$ for 24 hours. The studied samples were plates sized $3.5 \times 2.5 \times 6 \mathrm{~mm}$. The samples' mirror surfaces were prepared by mechanical polishing with diamond paste with subsequent recrystallization annealing and electropolishing (samples were polished up to 0-40 surface finish class). X-ray structure and phase analysis of $\mathrm{Co}-\mathrm{Cr}$ alloy samples has also been performed using a DRON-3.0 X-ray diffractometer.

Optical characteristics of mirror surfaces of $\mathrm{Co}-\mathrm{Cr}$ alloys with 10, 20 and 35\% chromium, as well as pure $\mathrm{Co}$ and $\mathrm{Cr}$, were investigated within the spectral range 0.23 to $2.8 \mu \mathrm{m}(0.44 \ldots 5.36 \mathrm{eV})$ using the original ellipsometer and the Beattie ellipsometric method [7]. The azimuth $\psi$ of the restored linear polarization and phase shift $\Delta$ between the $p$ - and $s$-polarized components of light were measured near the principal angle of 
incidence at various wavelengths; optical constants $n$ and $\kappa$ were then calculated as $n=\sin \varphi \tan \varphi \frac{\cos 2 \psi}{1+\sin 2 \psi \cos \Delta}$, $\kappa=\sin \varphi \tan \varphi \frac{\sin 2 \psi \sin \Delta}{1+\sin 2 \psi \cos \Delta}$, where $\varphi$ is the angle of incidence. Errors in determining the refractive and absorption indices $n$ and $\chi$ depended significantly on the spectral region in which measurements were carried out. Thus, in the ultraviolet, visible and near-infrared regions of spectrum they did not exceed $1.5 \%$, and in the infrared region they increased to $3-4 \%$. Based on the optical constants $n$ and $\kappa$, other optical characteristics were calculated, namely: dielectric permittivity $\varepsilon=n^{2}$ $\chi^{2}$, reflection coefficient $R=\frac{(n-1)^{2}+\chi^{2}}{(n+1)^{2}+\chi^{2}}$ at normal incidence and optical conductivity $\sigma=4 \pi \varepsilon_{0} n \chi v$ (where $v$ is the light frequency, $\varepsilon_{0}-$ vacuum dielectric permittivity), which is proportional to the interband density of electron states $G(h v)[8]$.

\section{Results and discussion}

Cobalt and chromium belong to the group of $3 d$ transition metals. Cobalt belongs to the eighth group of periodic table of elements, and chromium - to the sixth one. The difference in the number of $d$-electrons per atom affects significantly the properties of these metals. At temperatures below $700 \mathrm{~K}$, cobalt is characterized by a hexagonal closely packed (hcp) lattice ( $\varepsilon$-phase) and at higher temperatures - a face-centered cubic (fcc) lattice $(\gamma$-phase). Solid chromium has a body-centered cubic (bcc) lattice ( $\alpha$-phase) [9,10]. Chromium at temperatures below $311.5 \mathrm{~K}$ is a typical antiferromagnetic material, cobalt at temperatures below $1394 \mathrm{~K}$ is a ferromagnetic material (at higher temperatures both are paramagnetic metals). Therefore, the average magnetic moment per alloy atom decreases almost linearly (Slater-Pauling curve). According to phase diagrams [11], Co-Cr alloy with $10 \% \mathrm{Cr}$ is ferromagnetic, and $\mathrm{Co}-\mathrm{Cr}$ alloys with 20 and $35 \% \mathrm{Cr}$ show paramagnetic properties.

Analysis of the dependence of optical properties on the photon energy $h v$ in the studied spectral range $0.44 \ldots . .5 .09 \mathrm{eV}$ provides information about changes in the electronic structure of $\mathrm{Co}$, when $\mathrm{Cr}$ is added. At the first stage, a detailed study of the dispersion of the reflection coefficient $R$ and dielectric permittivity $\varepsilon$ within the above range was performed (Fig. 1).

Fig. 1 shows that the $R(h v)$ curves of all the studied alloys are similar to the curve of pure cobalt $(10 \% \mathrm{Cr}$ curve almost coincides with it). The $R$ value of all the alloys in the IR region $(h v<1 \mathrm{eV})$ reaches $0.75 \ldots 0.80$, and in the UV region $(h v>4 \mathrm{eV})$ is characterized by minimum absorption of pure $\mathrm{Co}$ and $\mathrm{Cr}$, the reflection coefficient $R$ also takes its minimum values. It should be noted that the maxima on the curves for Co-Cr with $20 \%$

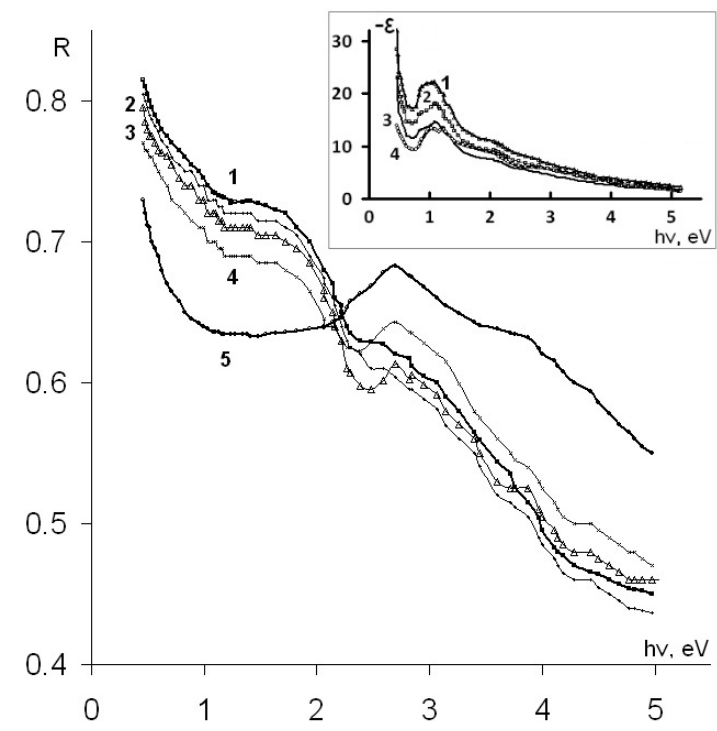

Fig. 1. Dispersion curves of the reflection coefficient $R(h v)$ for $\mathrm{Co}(1), \mathrm{Cr}(5)$ and their alloys with 10 (2), 20 (3) and $35 \%$ (4) chromium content. Inset shows the dielectric permittivity $\varepsilon(h v)$ dispersion curves for $\mathrm{Co}(1)$ and $\mathrm{Co}-\mathrm{Cr}$ alloys containing 10 (2), 20 (3) and 35\% (4) of Cr.

$\mathrm{Cr}$ and $35 \% \mathrm{Cr}$ practically coincide with the corresponding peak of pure chromium and are located at $2.85 \ldots 2.95 \mathrm{eV}$. It is noteworthy that with increase in the chromium concentration the intensity of this peak increases too, so we can assume that with its further increase the dispersion of alloy's reflection coefficient will become similar to the curve of pure chromium. Special attention should be given to the analysis of the dispersion curves $\varepsilon(h v)$ shown in Fig. 1 (inset). All the curves are characterized by normal dispersion at $h v=$ $1.1 \ldots 5.09 \mathrm{eV}$. The monotonous increase in the absolute value of dielectric permittivity $|\varepsilon|$ with the decrease of $h v$, typical of most metals, is observed in this spectral range. Anomalous dispersion is observed in the longwave region $h v=0.7 \ldots 1.09 \mathrm{eV}$ for the studied alloys, which indicates strong interband absorption in this spectral range. This assumption is supported by the results of the study of dispersion dependences for the optical conductivity $\sigma(h v)$ of Co-Cr alloys. The experimental curves $\sigma(h v)$ for the investigated alloys as well as for pure $\mathrm{Co}$ and $\mathrm{Cr}$ are shown in Fig. 2.

At first, let us briefly summarize characteristic properties of $\sigma(h v)$ spectra of pure Co and Cr. It can be seen that in the entire studied region $h v=0.44 \ldots .5 .09 \mathrm{eV}$ light absorption is caused by interband electron transitions for pure cobalt and chromium. The most characteristic cobalt spectrum distinction is a strong absorption band with fine structure and peaks at $h v=0.96$ and $1.28 \mathrm{eV}$. Weaker absorption bands appear at $h v=3.15 \ldots 3.25$ and 4.3...4.9 eV. Absorption in chromium is essentially different from that of cobalt. The $\sigma(h v)$ curve of chromium is characterized by a broad absorption peak at $1.8 \ldots 2.2 \mathrm{eV}$, which is in agreement with that shown in 


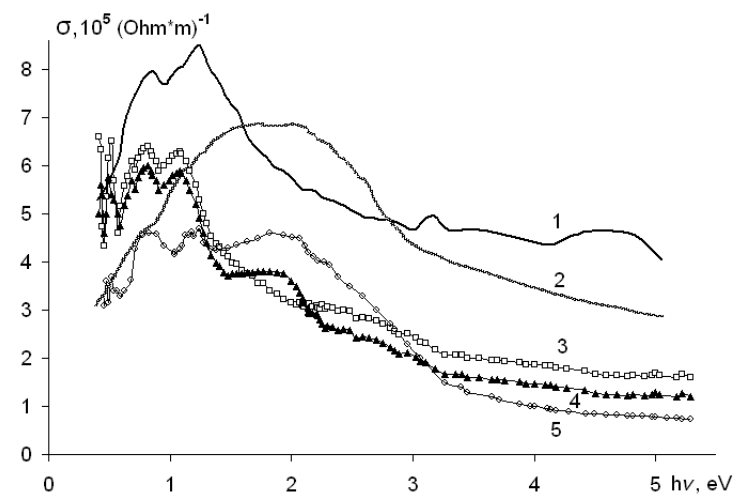

Fig. 2. Dispersion curves of the interband optical conductivity $\sigma(h v)$ for $\mathrm{Co}(1), \mathrm{Cr}(2)$ and their alloys with 10 (3), 20 (4) and 35\% (5) chromium content. Curves 1 and 2 for pure components are raised by $1 \cdot 10^{5}(\mathrm{Ohm} \cdot \mathrm{m})^{-1}$.

[9]. The intensity of the main $\sigma(h v)$ absorption peak decreases sharply, when $10 \%$ of $\mathrm{Cr}$ are added (Fig. 2), and both peaks at 0.96 and $1.28 \mathrm{eV}$ shift slightly (by approximately $0.15 \mathrm{eV}$ ) to the long-wave side of the spectrum. The main difference is the appearance of a new absorption band at $0.51 \mathrm{eV}$ and, maybe, at $2.1 \mathrm{eV}$ on the $\sigma(h v)$ curve of this alloy. When $20 \% \mathrm{Cr}$ are added, the intensity of both peaks decreases slightly, their position on the energy scale remains practically unchanged. It is seen from Fig. 2 that the $\sigma(h v)$ curve for Co-Cr alloy with 35\% $\mathrm{Cr}$ differs significantly both from $\sigma(h v)$ curves for pure Co and $\mathrm{Cr}$ and the $\sigma(h v)$ curves for Co-Cr alloys with 10 and $20 \%$ of $\mathrm{Cr}$. A single asymmetric band appears at $0.95 \mathrm{eV}$ instead of cobalt's dual structure, and a broad peak is present at $2.1 \mathrm{eV}$. The data obtained are indicative of the presence of cobalt's main absorption band at $h v=$ $0.8 \ldots 1.4 \mathrm{eV}$ in $\sigma(h v)$ curves for all the studied alloys, which indicates that the principal features of cobalt's band structure are preserved in alloys. However, an additional absorption band appears in $\sigma(h v)$ curves for all the studied alloys. These changes are associated with the restructuring of the energy spectrum of $\mathrm{Co}$ and the emergence of resonant impurity states [12].

Our analysis of $\sigma(h v)$ curves indicates that the optical spectra of the investigated alloys cannot be obtained by simple superposition of pure $\mathrm{Co}$ and $\mathrm{Cr}$ $\sigma(h v)$ spectra according to the mass ratios of these components. Additional optical absorption is present in Co-Cr alloys, which is associated with chromium impurities. The quantitative measure of this absorption is given by the value of the residual optical conductivity $\sigma(h v)$, which is the difference between the conductivity of the alloy $\sigma(h v)$ and that of pure Co $\sigma_{\mathrm{Co}}(h v)$, with its mass $(1-c)$ taken into consideration: $\Delta \sigma(h v)=\sigma(h v)-(1-c) \sigma_{\mathrm{Co}}(h v)$, where $c$ is the $\mathrm{Cr}$ concentration, $\sigma_{\mathrm{Co}}(h v)$ - optical conductivity of Co. Dispersion curves of the residual optical conductivity $\sigma(h v)$ are shown in Fig. 3 for the studied Co-Cr alloys.

Two additional absorption bands emerge with peaks at 0.55 and $1.85 \mathrm{eV}$ with the addition of $10 \%$ of chromium. Both bands' intensities increase monotonously and nonlinearly with increase in the $\mathrm{Cr}$ concentration. The short-wave band is more intensive; its peak, unlike the peak of long-wave band, shifts slightly into the short-wave direction with the increase in the $\mathrm{Cr}$ concentration. These results indicate that two impurity bands are formed in the electronic spectra of Co-Cr alloys, located respectively at $0.55 \ldots 0.56$ and $1.85 \ldots 2 \mathrm{eV}$ higher than the Fermi level $E_{\mathrm{F}}$. Lattice parameters change due to Co ions being substituted by $\mathrm{Cr}$ ions, which leads to distortion of Co host energy bands and their broadening as a result of decreased electron excitations lifetime. As it was said, the electronic spectrum of solid Co-Cr solutions is based on the pure Co electronic spectrum. The main features of absorption spectra are mainly associated with Van Hove critical points [8], which according to Co band structure [12] calculations are located around points $M, \Gamma$ and $H$ of the Brillouin zone [13]. Due to interband transitions at these points, main features of the $\sigma(h v)$ absorption spectrum for $\mathrm{Co}-10 \% \mathrm{Cr}$ alloy are formed in two band systems for electrons with different spin orientations (exchange splitting); the ferromagnetic fraction in the alloy was around $20 \%$, according to our calculations. It is strange, however, that the same features are present in the $\sigma(h v)$ spectrum of the $\mathrm{Co}-20 \% \mathrm{Cr}$ alloy, which according to [11] has to be paramagnetic. Our data show that at least in the skin layer the portion of the ferromagnetic phase in the $\mathrm{Co}-20 \% \mathrm{Cr}$ alloy is approximately the same as in the $\mathrm{Co}-10 \% \mathrm{Cr}$ alloy. The above structure is absent in $\sigma(h v)$ spectrum of $\mathrm{Co}-35 \% \mathrm{Cr}$ alloy, as it is paramagnetic. Thus, the electronic spectra of Co-Cr solid solutions with $\mathrm{Cr}$ concentrations up to at least $35 \%$ are pure Co electronic spectra, deformed and broadened due to electron excitation lifetime decrease, with the exchange interaction energy around $0.5 \mathrm{eV}$ according to obtained data [12] $(\mathrm{Co}-10 \% \mathrm{Cr}$ and $\mathrm{Co}-$ $20 \% \mathrm{Cr}$ alloys). Absence of exchange interaction in $\mathrm{Co}-$ $35 \% \mathrm{Cr}$ alloy leads to broadening the main absorption

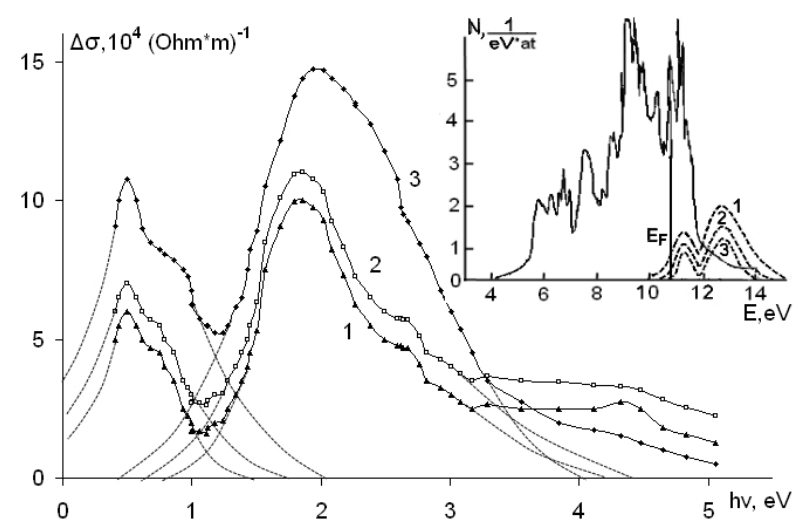

Fig. 3. Dispersion of residual optical conductivity $\Delta \sigma(h v)$ for Co-Cr alloys containing $10(1), 20(2)$ and $35 \%$ (3) of $\mathrm{Cr}$. Inset is the curve of density of states for pure Co [12]. Dashed line is the curve of density of states for Co-Cr alloys with $10(1), 20$ (2) and 35\% (3) of Cr. The vertical solid line is the Fermi level energy $E_{\mathrm{F}}$. 
band in the optical conductivity spectrum. Also, an additional band is formed by chromium impurity, with the maximum density of electron states, which is approximately at $1.85 \ldots 2.0$ and $0.55 \ldots 0.56 \mathrm{eV}$ above the Fermi level $E_{\mathrm{F}}$.

If it is assumed that the new absorption band is formed due to electron transitions from Co host states near the Fermi level $E_{\mathrm{F}}$ into the free states of the impurity band, as in other binary transition metal alloys, such as $\mathrm{Ni}-\mathrm{Fe}, \mathrm{Ni}-\mathrm{Cr}$ and Fe-Co [8], than being based on experimental data it is possible to determine the density of states $N(E)$ of the impurity band [8]. To calculate $N(E)$ of a certain alloy, it is necessary to find the energy distance between the centre of the impurity band and the Fermi level $E_{d}-E_{\mathrm{F}}$ and its halfwidth $\Delta[8]$ according to the experimental data. Experimental $E_{d}-E_{\mathrm{F}}$ and $\Delta$ values, according to Fig. 3 data, are respectively 1.85 and $1.31 \mathrm{eV}$ (Co-10\%Cr alloy), 1.91 and $1.42 \mathrm{eV}$ (Co-20\%Cr alloy), 2.0 and $1.52 \mathrm{eV}(\mathrm{Co}-35 \% \mathrm{Cr}$ alloy) for the main band. For the additional band, these values are respectively 0.55 and $0.71 \mathrm{eV}(\mathrm{Co}-10 \% \mathrm{Cr}$ alloy), 0.55 and $0.79 \mathrm{eV}$ (Co-20\% $\mathrm{Cr}$ alloy), 0.56 and $0.79 \mathrm{eV}(\mathrm{Co}-35 \% \mathrm{Cr}$ alloy). The $N(E)$ curves shown with the dashed lines in Fig. 3 were calculated for all the studied alloys by using the $E_{d}-E_{\mathrm{F}}$ and $\Delta$ values for the main absorption band.

The density of states in any alloy can be approximately considered as superposition of the density of states for Co host with a certain weight equal to the Co concentration in the alloy and the density of states $N(E)$ within the impurity band. As to the low-energy absorption band (Fig. 3), it can be considered as follows. It is unlikely that it is associated with an impurity band split due to exchange interaction, which is the largest among all ferromagnetic and antiferromagnetic metals for $\mathrm{Cr}$ [14], as this long-wave band is present in $\mathrm{Co}-35 \% \mathrm{Cr}$ alloy, which unlike $\mathrm{Co}-10 \% \mathrm{Cr}$ and $\mathrm{Co}-20 \% \mathrm{Cr}$ alloys is paramagnetic. At the same time, in the spectrum of the residual conductivity $\sigma(h v)$ in the alloy $\mathrm{Co}-35 \% \mathrm{Cr}$ the indicated band is observed, though this alloy, unlike alloys Co- $10 \%$ $\mathrm{Cr}$ and $\mathrm{Co}-20 \% \mathrm{Cr}$, is in the paramagnetic state, and it is clear that the exchange splitting in this case is absent. The intensity of this low-energy band is also approximately thrice lower than the main short-wave band (Fig. 3). One of the other possible causes of this band may be formation of a new band due to vacancies in the anion sublattice of $\mathrm{Co}-\mathrm{Cr}$ solid solutions, the concentration of which increases slightly with increasing the impurity content of Cr. A definite origin of the long-wave absorption band can be found from temperature dependences of the optical properties of $\mathrm{Co}-\mathrm{Cr}$ solid solutions with a small concentration of $\mathrm{Cr}$ in a wide temperature range, including the Curie point of studied alloys.

\section{Conclusions}

It has been found that new impurity bands are formed in the electronic spectrum of host $\mathrm{Co}$, when $\mathrm{Cr}$ impurities are added, which occur in the infrared spectral range and are responsible for additional absorption in these alloys. Principal features of the host Co electronic spectrum are preserved in the studied alloys' spectra. It has been shown that the electronic spectra of $\mathrm{Co}-\mathrm{Cr}$ alloys are the superposition of densities of electron states $N(E)$ of pure cobalt with a certain weight and the density of states of chromium within the impurity band.

\section{References}

1. A.O. Animalu, Electronic structure of transition metals. Quantum defects and model potential // Phys. Rev. 8(3), p. 354-358 (1993).

2. S. Wang, Cobalt - its recovery, recycling, and application // JOM, 58(10), p. 47-50 (2006).

3. J. Izquierdo and C. Demangeat, Magnetic configurations of a Co monolayer on $\mathrm{Cr}$ substrates // Phys. Rev. 62(9), p. 1228-1232 (2000).

4. M.B. Stearns, C.H. Lee, and T.L. Groy, Structural studies of $\mathrm{Co} / \mathrm{Cr}$ multilayered thin films // Phys. Rev. 40(3), p. 8256 (1989).

5. P. Franke, D. Neuschütz, Binary systems. Part 2: Elements and binary systems from B-C to $\mathrm{Cr}-\mathrm{Zr} / /$ J. Phys. Chem. 19(2), p. 1-5 (2004).

6. T. Mukherjee, S. Sahoo, R. Skomski, D.J. Sellmyer, and $\mathrm{Ch}$. Binek, Magnetocaloric properties of $\mathrm{Co} / \mathrm{Cr}$ superlattices // Phys. Rev. 79(4), p. 144-146 (2009).

7. H. Fujiwara, Spectroscopic Ellipsometry. Principles and Applications. Atrium, Wiley, NY, 2007, p. 324-357.

8. L.V. Poperenko, V.S. Stashchuk, I.A. Shaikevych, V.A. Odarych, Diagnostics of Surface with Polarized Light. Publishing Center "Kyiv University", Kyiv, p. 130-136 (2007)

9. Y.J. Choi, J. Okamoto, D.J. Huang, K.S. Chao, Thermally or magnetically induced polarization reversal in the multiferroic $\mathrm{CoCr}_{2} \mathrm{O}_{4} / /$ Phys. Rev. Lett. 10(2), 067601 (2009).

10. U.K. Rößler, A.N. Bogdanov, Reorientation in antiferromagnetic multilayers spin-flop transition and surface effects // Phys. Rev. 69(2), p. 184-192 (2004).

11. H. Okamoto, Phase diagram of Co-Cr (CobaltChromium) // J. Phase Equilibria, 24(4), p. $377-$ 378 (2003).

12. N.I. Kulikov, E.T. Kulatov, Electronic band structure, Fermi surface and optical properties of cobalt under pressure // J. Phys. 12(7), p. 2267 2289 (1982).

13. V.S. Stashchuk, O.P. Polianska, S.I. Stashenko, Optical and electronic properties of metallic cobalt in different structural states // Ukrain. J. Phys. 55(4), p. 389-393 (2010).

14. S.V. Vonsovskii, Magnetism, Magnetic Properties of Dia-, Para-, Ferro-, Antiferromagnetics, and Ferrimagnetics. Nauka, Moscow, 1971, p. 186-191 (in Russian). 